\title{
In-Hospital Respiratory Viral Infections for Patients with Established BPD in the SARS-CoV-2 Era
}

\author{
Matthew Kielt ${ }^{1}$, Angela Murphy ${ }^{1}$, Jodi Smathers ${ }^{1}$, Maleah Bates ${ }^{1}$, Leif Nelin ${ }^{1}$, and Edward \\ Shepherd ${ }^{2}$
}

${ }^{1}$ Nationwide Children's Hospital

${ }^{2}$ Nationwide Childrens Hospital

August 3, 2021

\begin{abstract}
Objective Our objective was to test the hypothesis that in-hospital respiratory viral infections (RVI) would be significantly lower in a cohort of patients with established bronchopulmonary dysplasia in the SARS-CoV-2 era when compared to historical controls. Study Design On April 1, 2020, we implemented a universal infection prevention bundle to minimize the risk of nosocomial SARS-CoV-2 transmission in a dedicated BPD intensive care unit. We performed a retrospective cohort study and included patients with established BPD, as defined by the 2019 Neonatal Research Network criteria, admitted to our center who underwent real-time polymerase-chain-reaction RVI testing between January 1, 2015 and March 31, 2021. We excluded patients re-admitted from home. We compared to number of tests performed, the proportion of positive tests, and the distribution of viral respiratory pathogens in the pre- and post-SARS-CoV-2 eras. Results Among 176 patients included in the sudy, 663 RVI tests were performed and $172(26 \%)$ tests were positive. The median number of tests performed, measured in tests per patient per month, in the SARS-CoV-2 era was not significantly different compared to the pre-SARS-CoV-2 era (0.45 vs 0.34 tests per patient per month, $\mathrm{P}=0.07)$. The proportion of positive RVI tests was significantly lower in the SARS-CoV-2 era when compared to the pre-SARS-CoV-2 era $(0.06$ vs $0.30, \mathrm{P}<0.0001)$. No patients tested positive for SARS-CoV-2 in the SARS-CoV-2 era. Conclusions Infection prevention measures developed in response to the SARS-CoV-2 pandemic may reduce the risk of RVIs in hospitalized patients with established BPD.
\end{abstract}

\section{Hosted file}

In-Hospital VRI for BPD in SARS-CoV-2 final (2).docx available at https://authorea.com/ users/428948/articles/532735-in-hospital-respiratory-viral-infections-for-patients-withestablished-bpd-in-the-sars-cov-2-era 


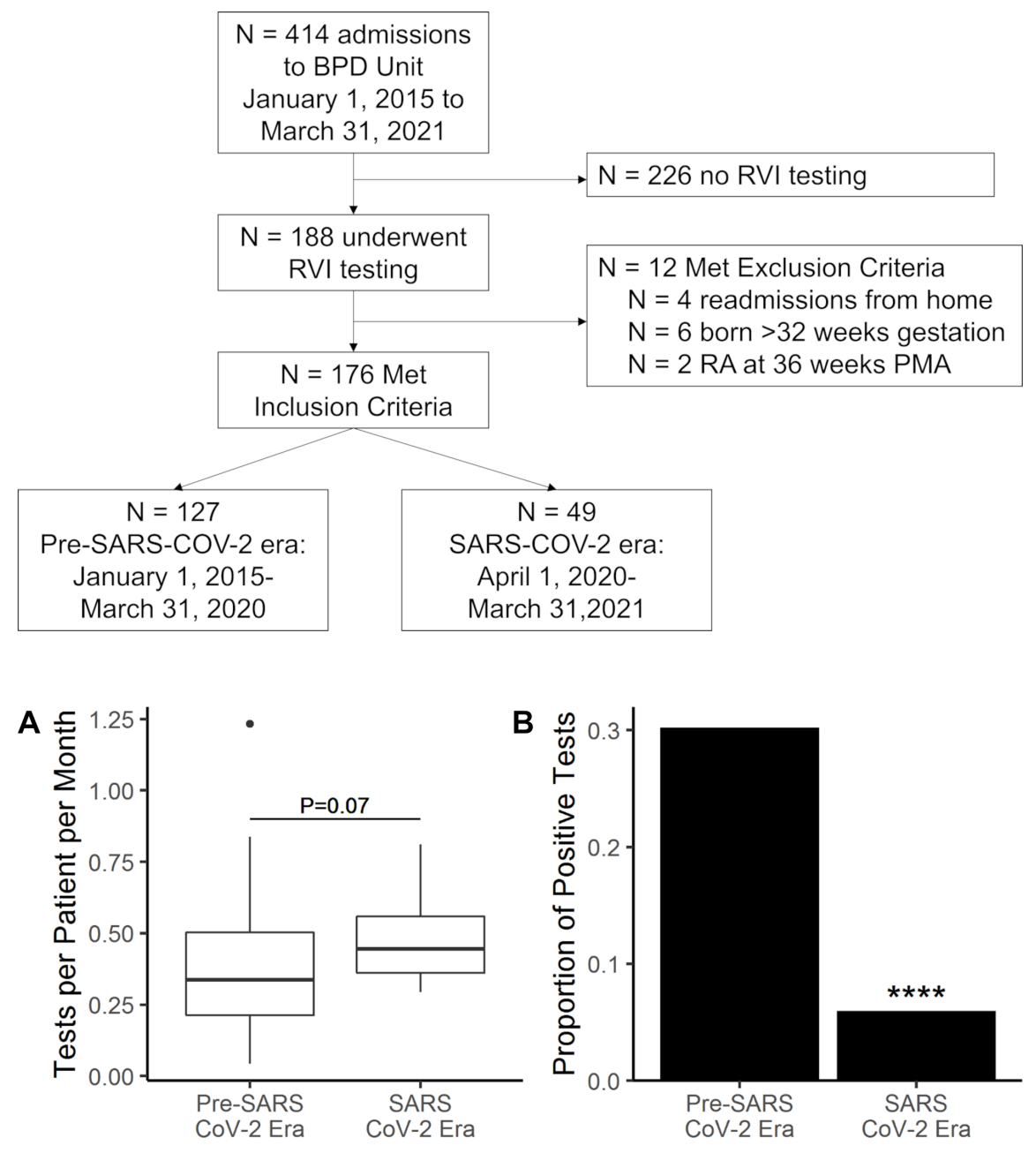

